\title{
Event Detection in Time Series by Genetic Programming
}

\author{
Feng Xie \\ School of Computer Science and IT \\ RMIT University, Australia \\ feng.xie@ rmit.edu.au
}

\author{
Andy Song \\ School of Computer Science and IT \\ RMIT University, Australia \\ andy.song@rmit.edu.au
}

\author{
Vic Ciesielski \\ School of Computer Science and IT \\ RMIT University, Australia \\ vic.ciesielski@ rmit.edu.au
}

\begin{abstract}
The aim of event detection in time series is to identify particular occurrences of user-interest in one or more time lines, such as finding an anomaly in electrocardiograms or reporting a sudden variation of voltage in a power supply. Current methods are not adequate for detecting certain kinds of events without any domain knowledge. Therefore, we propose a Genetic Programming (GP) based event detection methodology in which solutions can be built from raw time series data. The framework is applied to five synthetic data sets and one real world application. The experimental results show that working on raw data even with a dimensionality as high as $140 \times 80$, genetic programming can achieve superior performance to conventional methods operating on pre-defined features. Furthermore, analysis of the evolved event detectors shows that they have captured the regularities inserted into the synthetic data sets and some individuals can be readily understood by humans.
\end{abstract}

Index Terms-genetic programming; feature extraction; event detection; time series.

\section{INTRODUCTION}

A time series is a sequence of data points which are the states of a single variable or multiple variables over a period of time. An event in a time series is the occurrence of a variation in values over a time span that is of particular interest to a user. For example the occurrence of an inactive or even flat electrocardiogram would be an alarming event for medical practitioners or a sudden change of voltage in a power supply may require the attention of maintenance operators. The above two examples only involve a single variable. In many circumstances, an event may be signified by simultaneous variations occurring in multiple variables. For example a certain heart condition may be diagnosed not only by the electrocardiogram but also by blood pressure values and other measurements at the same point of time. Another example of multiple variables is electroencephalography (EEG). The signals are acquired through multiple sensors on the scalp, each recording the electrical activities for one region of the brain. In some multivariable time series, the variables are spatially dependent. Video is such an example. Changes in a single pixel are not very meaningful and likely be noise rather than a video event which often occurs in neighbouring pixels. A video event could be appearance of an object, movement of an object or a change of scene. In these three examples, a single variable is not sufficient for identifying an event. Features that combine multiple variables or detection methods that can handle simultaneous variations in multiple variables are essential for such applications. Another issue in event detection is the time span of an event. An event may appear within two consecutive sampling points such as a sudden change in voltage or may last for hours or even days such as elevated temperatures in a heat wave. The span of an event varies significantly from problem to problem. Determining the duration of an event and devising features accordingly often require extensive domain knowledge from human experts.

There is a considerable published literature on time series prediction where the aim is to forecast future unknown values. There are many fewer publications on classifying time series, discovering events and recognising temporal patterns. However, interest in this area has increased recently. Recent work seems to be moving in two directions. One direction is extracting all features that can define an event and then detecting the presence of the event by a pattern matcher [15][19]. Such a technique is only suitable when rules for determining the occurrence of an event are clear, including the time span of the event and its defining characteristics. However, when the events of interest are not well-understood or involve irregularities, this approach will not perform well. Moreover the solution for one problem is unlikely to be suitable for other problems as the rules are highly problem dependent. The other direction, which is different from this matchingextracted-features method, is to detect change points[14][16]. The basic assumption of this approach is that any subsequence of a time series can be modelled mathematically. The models are characterized by a set of parameters. Any statistically significant change in the parameters, or the model itself, is then considered as an event. This approach requires no priori knowledge as the model is constructed from the data and is generally applicable to various domains without much human intervention. However, this approach is not appropriate for applications where the events are characterised by complex relationships between variables, for example the multi-variable variations in electrocardiograms and EEG signals mentioned earlier. A framework which can combine the benefits of these two approaches is highly desirable. Ideally, using such a method we can automatically generate features or decision rules for differentiating events of user interest from the background, either in a single variable or in multiple variables. Domain knowledge or pre-defined features would not be 
mandatory and the method would not be limited to just detecting changes in variable values but could recognize various events involving complex relationships between variables. This is the main aim of the Genetic Programming (GP) [1] based methodology proposed in this study.

For the purposes of this work, we consider an event to be variations of a single variable or multiple variables during a time span of interest to the user. An event can be as simple as a change in one variable or as complex as a large change in any two out of five variables, or even a large change in groups of pixels on video frames. The time series we use in this study is a labelled time series stream in which the label indicates whether the event of interest has just occurred. The evolutionary process must determine how many prior data points are needed to accurately detect the event. The main goal of this paper is to develop a GP based framework for detection of events in such a time series stream. The specific questions addressed in this investigation are:

1) How can the event detection problem be represented in GP so that detectors based on raw data points can be evolved?

2) How does the GP based approach compare to conventional feature based approaches on a range of tasks?

3) Can a human understandable description of an event be extracted from the evolved GP detectors?

We will evaluate the performance of the proposed framework on a number of detection problems of increasing difficulty. The problems use five synthetic data streams and a data stream from a real world application. The conventional methods are the OneR rule based classifier, the J48 decision tree classifier, the Naïve Bayes classifier, the IB1 nearest neighbour classifier and the AdaBoost meta classifier, all from the Weka machine learning system.

\section{BACKGROUND AND RELATED WORK}

There are existing studies applying GP to time series data. Most of these are for forecasting. Kaboudan used GP for the purpose of housing price prediction, taking annual median price of single family homes of 12 neighbouring areas in Cambridge as temporal-spatial input. They found that the results produced by GP were more reliable and logically acceptable than those from neural networks [4]. Wagner and Michalewicz proposed GP with adaptive windowing for forecasting in a dynamic environment [7]. Song and Pinto applied GP video frames for motion detection problems [5]. This study was based on pixel values where the evolved GP detectors operated on points rather than regions. However they were able to distinguish regions of interesting movements from regions of non interesting movements. Hetland et.al combined a pattern match chip and GP to discover temporal rules which were used for prediction [6]. These rules relied on previous values on the time series. Additional hardware devices and additional time are needed to set up the entire system. Furthermore it does not work well on more complex real world data. Although these works have a different goal to ours, they do show the potential of GP for handling time series data and finding rules.
Most event detection algorithms require many parameters to describe an event. Keogh et.al. [17] proposed an anomaly detection method which only requires one parameter from the domain expert to determine a time series anomaly, the length of the event. A fixed-length sliding window then moves through the entire time series. All possible subsequences are examined to find anomalies which are subsequences with some user defined properties. However, such a fixed-length window is only sensible if the characteristics of anomalies are consistent and are known. In problems where the event span time is unknown and the occurrence of an event is unpredictable, a flexible window size will be essential. Effectively no pre-defined parameter should be introduced for this type of circumstances. Our GP approach aims to address this issue.

Our work is also related to time series classification [18][21]. Time series classification assigns a time series to one of two or more predefined classes. This is similar to our approach in which events of interest are positives and other events or background signals are negatives. Like existing work on pattern-matching and anomaly detection, time series classification often requires domain knowledge for feature extraction and for determining time intervals between patterns. The GP method presented in this study aims to eliminate such a requirement and to be independent of problem domains.

\section{GP REPRESENTATIONS}

Since the majority of real world time series sequences are real-valued, we establish a primary set of GP focusing on numeric data. As well as presenting the function set which is in Table I and the terminal set which is in Table III, our discussion on the GP representation in this section also includes the ways of handling temporal relationships which is crucial in processing time series data. The general discussion on it is in Section III-A, while the representation for handling multiple variables is described in Section III-B and that for handling multiple variables with additional spatial relationships is described in Section III-C.

TABLE I

FUNCTION SET OF GP

\begin{tabular}{l|l|l}
\hline \hline Function & Parameters & Return Type \\
\hline+ & Double, Double & Double \\
+ & Double, Double & Double \\
$*$ & Double, Double & Double \\
$/$ & Double, Double & Double \\
\hline Temporal_Diff & Double i & Double \\
\hline \multirow{2}{*}{ Window } & $\begin{array}{l}\text { Double i, } \\
\text { Int operation, } \\
\text { Int temporal-index }\end{array}$ & Double \\
\hline \multirow{2}{*}{ Multi-Variable } & $\begin{array}{l}\text { Int multivariable-operation, } \\
\text { Int variable-index }\end{array}$ & Double \\
\hline \multirow{2}{*}{ Region } & $\begin{array}{l}\text { Int multivariable-operation, } \\
\text { Vector coordinate, }\end{array}$ & Double \\
& Vector area & \\
\hline
\end{tabular}

\section{A. General Representation}

There are special characteristics existing in time series data, which record the states of one variable at every sampling point 
along the time line. The order of these points is immutable and the series is only one directional, from early to present. Points that are closer to a point have more influence on that point. To capture these characteristics, we use a sliding window to include the current value and several of its immediate preceding values. Sliding window is used in other studies on time series data [17]. The mechanism of sliding window is illustrated in Figure 1. GP is expected to define a suitable window size for each problem. Furthermore, a GP program may not use all the points in the window, but a selection of them.

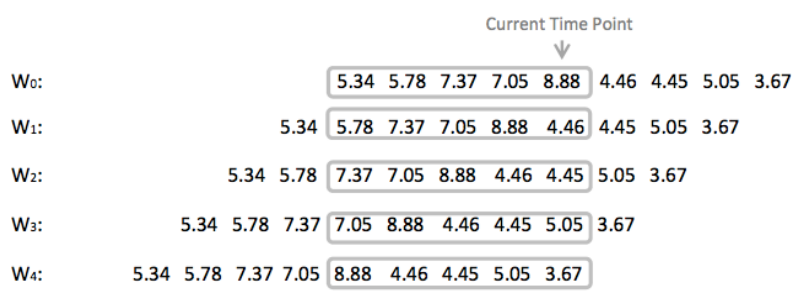

Fig. 1. Example of A Sliding Window (Size $=5)$ Moving on A Time Series

1) Temporal_Diff Function: Temporal change between adjacent points is obviously important for identifying the occurrence of events. So we introduce such a function, "Temporal_Diff", as one of the fundamental operators. It takes one double value as its input $i$ and calculates the difference between points $t_{i}$ and $t_{i-1}$. Effectively this function operates on a sliding window of size 2 .

2) Window Function: This "Window" function is the main function for defining the input, selecting data points inside the window, and applying the operations on the select data points. It takes three parameters: $i$, temporal index and operation. The first one is the input of this function which samples a data point at every time step. It keeps the subsequence of historical values of that input. The length of this subsequence is adjustable, which is set as 8 in this study. These data points are marked from the earliest point to the most recent one as $t_{0}, t_{1}, \ldots, t_{S-1}$, where $S$ is the window size. The second parameter is from terminal "Temporal Index" which returns an random integer within the range of $\left[1,2^{S}-1\right]$. This integer is converted into its binary form. This binary is mapped to the subsequence of time series data under the window. A bit with "1" indicated the data point with the same index will be selected.

TABLE II

EXAMPles of DATA POINTS SELECTED By BINARY “TEMPORAL INDEX”

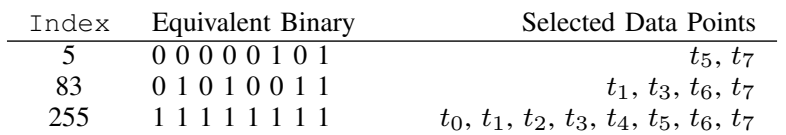

Table II illustrates this mapping along with three examples. The first one shows that when when 5 is the second input, then a "Window" function with a window size 8 will be guided by the binary form of 5 , which is 00000101 , to select points $t_{5}$ and $t_{7}$, since the sixth and the eighth bits contain 1 . The other two examples follow the same rule. This mechanism aims to improve the flexibility in point selection as it allows small subsections and a group of isolated points inside of a window.

The third parameter is a randomly generated integer valued from a range $[1,4]$. Each value corresponds to one of the four temporal operations: AVG, STD, DIF and SKEWNESS. They are used for calculating the average, the standard deviation, sum of differences and skewness of the selected points under the window. Its return value is then the output of the "Window" function. For illustration purpose this function is applied to the time series example from Figure 1, given the 2nd and 3rd parameters are 3 and AVG. Figure 2 shows this process which selects the 4th and 5th points at each window position since the binary form of 3 is 00011 for a 5 bit window. The output is the average of the two selected points.

\begin{tabular}{|c|c|c|c|c|c|c|c|c|c|c|c|}
\hline & \multicolumn{3}{|c|}{ Window } & \multicolumn{2}{|c|}{$\begin{array}{l}\text { temporal index: } 3 \\
\text { binary string: } 00011\end{array}$} & \multicolumn{2}{|c|}{ Selected } & Values & \multicolumn{2}{|r|}{ operation: AVG } & Result \\
\hline Wo: 5.34 & 5.78 & 7.37 & 7.05 & 8.88 & 5.34 & 5.78 & 7.37 & 77.05 & 8.88 & & 7.965 \\
\hline$W_{1}: 5.78$ & 7.37 & 7.05 & 8.88 & 4.46 & 5.78 & 7.37 & 7.05 & 58.88 & 4.46 & & 6.67 \\
\hline$W_{2}: 7.37$ & 7.05 & 8.88 & 4.46 & 4.45 & 7.37 & 7.05 & 8.88 & 84.46 & 4.45 & & 4.455 \\
\hline$W_{3}: 7.05$ & 8.88 & 4.46 & 4.45 & 5.05 & 7.05 & 8.88 & 4.46 & $\begin{array}{ll}6 & 4.45\end{array}$ & 5.05 & & 4.75 \\
\hline$N_{4}: 8.88$ & 4.46 & 4.45 & 5.05 & 3.67 & 8.88 & 4.46 & 4.45 & 5.05 & 3.67 & & 4.3 \\
\hline
\end{tabular}

Fig. 2. Example of Applying the "Window" Function (Size = 5)

\section{B. Functions for Multivariable Time Series}

It is not difficult to see that one "Window" function mentioned above only handles one variable changing over time. Events occurring on multiple variables would not be captured by it. So function "Multi-Variable" is introduced here to address that. It selects arbitrary collection of variables and computes characteristics of these variables. It takes two integers as its parameters: variable index and multivariable operation. There is no input parameter as all variables in the problem are treated as input and they selected by variable index. This parameter works in a similar way as to temporal index in the "Window" function, except its range is from 1 to $2^{M}-1$, where $\mathrm{M}$ is the total number of variables. So a binary form of 13,1101 , would tell the function to operate on the first, the second and the fourth variables. The parameter for multivariable operation also returns an integer from 1 to 4 , which corresponds to the following functions: median value which is the middle value of the selected variables (MED), their average (AVG), their standard derivation (STD) and the distance between the maximum and minimum values (RANGE). Note these operations are cross variables and the temporal operations in Section III-C are for data points of the same variable. Therefore they are different.

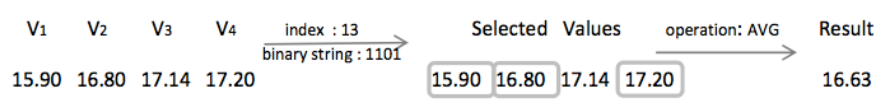

Fig. 3. Multi-Variable Function Operating on One Four-variable Time Series 
Figure3 illustrates how this "Multi-Variable" function operating on a four-variable time series $\left(V_{1}\right.$ to $\left.V_{4}\right)$ if its parameters are 13 and AVG. The average of $V_{1}, V_{2}$ and $V_{4}$ is returned as the function output. The "window" function can be integrated with "Multi-Variable" by taken the output of the latter as input data of the former. This combination enable GP to find both temporal relationships and variable dependence simultaneously.

\section{Functions for Spatially Related Multivariable Time Series}

Spatial dependence may exist in multivariable time series where some of the adjacent variables are related to each other. Videos can be considered as an example because it can be viewed as a matrix progressing along a time line. The neighbouring pixels on a frame are likely to influence each other. To handle this situation we introduce another new function "Region" to capture inter-variable dependence. It takes three parameters: Terminal multivariable operation, Terminal coordinate and Terminal area. One of four types of operations which are discussed in function "Multi-Variable" can be chosen, and then applied on selected points. The second parameter takes input from Terminal coordinate which provides a vector to specify the $\mathrm{x}$, $\mathrm{y}$ coordinates of top left corner of a region. The third parameter takes input from Terminal area to specify the width and the height of the selected region.

\begin{tabular}{|c|c|c|c|c|c|c|c|c|c|c|}
\hline \multicolumn{4}{|c|}{$V_{1}-V_{20}$} & \multirow{2}{*}{$\underset{\text { coordinate }:(1,2)}{\text { area }:(2,3)}$} & \multicolumn{2}{|c|}{ Selected } & \multicolumn{2}{|l|}{ Values } & \multirow[t]{2}{*}{ operation: AVG } & Result \\
\hline 33.99 & 38.64 & 37.14 & 38.36 & & 33.99 & 38.64 & 37.14 & 38.36 & & 16.63 \\
\hline 34.96 & 43.75 & 47.59 & 44.90 & & 34.96 & 43.75 & 47.59 & 44.90 & & \\
\hline 36.55 & 36.61 & 37.14 & 31.57 & & 36.55 & 36.61 & 37.14 & 31.57 & & \\
\hline 35.75 & 41.40 & 38.56 & 44.46 & & 35.75 & 41.40 & 38.56 & 44.46 & & \\
\hline 43.29 & 33.46 & 42.70 & 39.75 & & 43.29 & 33.46 & 42.70 & 39.75 & & \\
\hline
\end{tabular}

Fig. 4. Region Function Operating on a $(5 \times 4)$ Multivariable Time Series

This "Region" function enables GP to operate on an entire area rather than on a group of isolated points. Figure 4 illustrates its behaviour. Suppose the operation is AVG, the $x$ and $y$ coordinates are $(1,2)$ and area is $(2,3)$, then the average on the selected six points will be returned as the function output.

\section{Terminals}

Table III lists the terminals used in our GP representation. The first two are for general functions while the rest can only attach to certain functions as specified in Table I.

\section{TIme SERIES DATA AND Events}

A collection of six tasks with increasing level of difficulties are used to evaluate the GP method. They consist of both synthetic problems and real world application. Their event size can be different. While some contain only one variable, some are multivariable tasks. These artificial data sets are generated as thus: first a sequence of background noise containing no target event is generated; then some data points are randomly picked up and replaced with one or more events. The last one is video data which represents spatially dependent multivariable problems. The video for training and testing are clips from two different TV programs. There is no content similarity between the two.

\section{A. Single-Variable Time Series}

There is no need to consider variable dependence in this set of data. However the suitable window still varies from problem to problem.

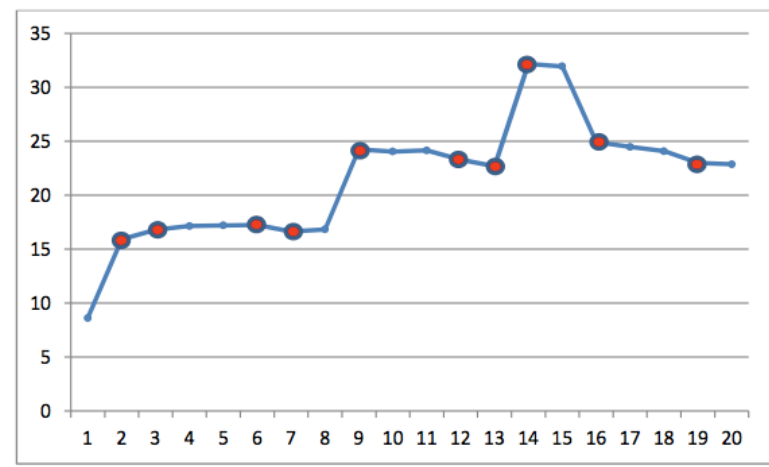

Fig. 5. Single Point Change with Tolerance $[-0.5,0.5]$

1) Single Point Changes: In many applications small changes in a variable are ignored unless the variation is beyond a certain range. A hyper-sensitive detector would be equally bad as an insensitive one if not worse. This task here simulates that need of a noise tolerant detector. So two thresholds are introduced to define a range of variation. The difference between two consecutive points should be considered as negative if it is within the range. Otherwise the variation should be reported as an event.

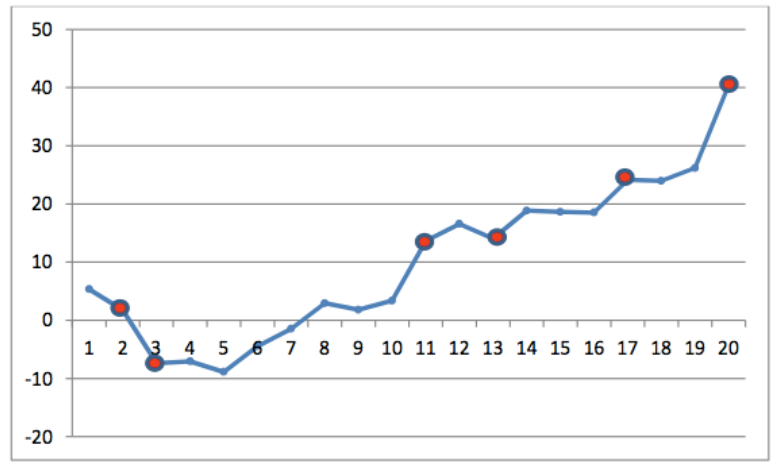

Fig. 6. Single Point Change with Tolerance $[-2.5,5.0]$

Two tasks with different tolerance are used for evaluation. Sample sequences are shown in Figure 5 and Figure 6. The tolerable variation between two consecutive points for the first task is in the range of $[-0.5,0.5]$, while that for the second task is much wider, $[-2.5,5.0]$. The positive points, e.g. the events, are highlighted with red dots on the figures. We can see that small changes are ignored on Figure 5, while on Figure 6 even some noticeable changes are not marked. 
TABLE III

TERMINAL SET

\begin{tabular}{l|c|c|l}
\hline Terminal & Value & Return Type & Function \\
\hline \hline Variable $m$ & Current value of Variable $m$ & Double & General \\
Random & A random value & Double & General \\
Operation & AVG,STD,DIF,SKEWNESS & Integer & Window \\
Temporal Index & {$\left[1,2^{\text {window-size }-1]}\right.$} & Integer & Window \\
Variable Index & {$\left[1,2^{\text {num-of-variables }}-1\right]$} & Integer & Multi-Variable \\
Multivariable Operation & MED,AVG,STD,RANGE & Integer & Multi-Variable, Region \\
Area & (width,height) & Width and height of an area & Region \\
Coordinates & $(\mathrm{x}, \mathrm{y})$ & Coordinate of a point & Region \\
\hline
\end{tabular}

For conventional methods, the feature for these events would be simply the difference between two adjacent data points as $t_{i}-t_{i-1}$.

2) Perturbed Periodical Function: The target events in some real world applications are not as simple as single point changes, but involve many data points. Furthermore the event would not be static but in periodical changes. So the variation in a true event would be much more complex. Here we introduce a periodical function $y=|100 * \sin (x)|$ which is sampled every $\frac{2 \pi}{7}$. Some data points are randomly replaced by values between 0 and 100 . A complete cycle at any phase is considered as an event, of which the size is eight. In other words, any eight consecutive sampled points of this function should be regarded as events. Any uncompleted circles or perturbation should be reported as negatives. Figure 7 illustrates a sample sequence on which two positives are marked by grey dash lines, differing from each other in shapes.

One feature is extracted for conventional classifiers by equation $\sum_{i=1}^{8}\left|t_{i}-t_{i-1}\right|$. The phases at which the events start have no effect on this feature, therefore even the two cycles in the figure look different, they have the identical feature values.

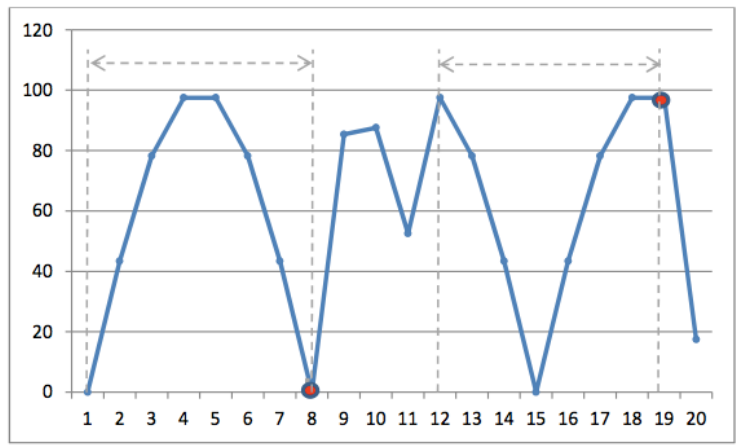

Fig. 7. Perturbed Periodical Function

\section{B. Multivariable Time Series}

1) Two Multivariable Tasks: In the case of multivariable time series, GP needs to identify which variables are related as any two could be dependent on each other. Besides the characteristics of this dependence could define the occurrence of an event. Two such tasks are introduced here. The first is shown in Figure 8, the event happens when there are significant simultaneous changes in both variables in this two-variable time series. The second is a more complex

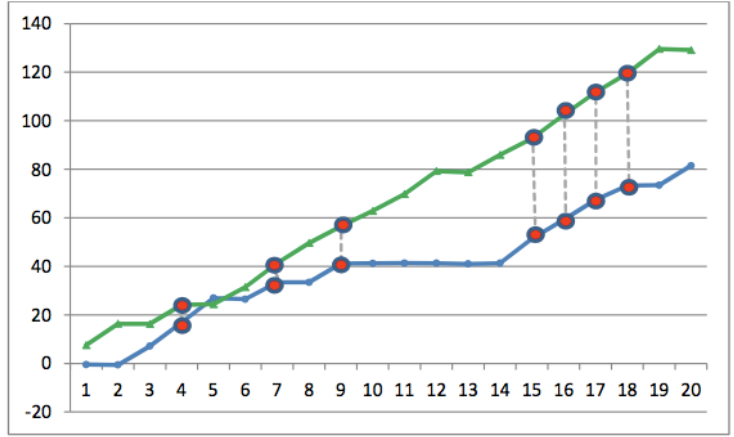

Fig. 8. Multivariable Time Series(Two Variables)

version which has five variables. If any two variables have simultaneous changes which are more than 5 , then an event should be reported. Similar to single variable tasks, these two data sets are randomly generated.

For this task, the pre-defined features are the differences between two adjacent points by $V_{m}\left(t_{i}\right)-V_{m}\left(t_{i-1}\right)$.

2) Spatially Dependent Multivariable Time Series: This task is to recognize scene changes on video clips. It is much more challenging compared to the previous five tasks. One reason is the high dimensionality of data as there are $140 \times 80$ pixels in a video frame. Additionally some pixels might be noisy. A group of features for conventional methods are extracted for this data. They are the differences between two consecutive frames on average intensity value, average Red, Green and Blue values.

\section{EXPERIMENTS AND RESULTS}

In our experiments, a standard GP setting was used. The parameters are listed in Table IV. The maximum tree depth is set as small as 5 to facilitate the following analysis of evolved programs. Also we did not find serious performance deterioration caused by this setting. Additionally, dynamic range selection which is proposed for GP to process classification problems was used in this study [23]. For every data set, the GP runs were conducted for ten times. The best among these ten runs was taken as outcome of GP. The details of the training and test data sets can be seen in first four columns of Table V. Most of the data sets are balanced.

For comparison purposes four popular conventional classifiers were applied onto all the six tasks, OneR [9], IBI [12]; Naïve Bayes [11] and J48 [10]. In addition AdaBoost was 
TABLE V

TRAINING AND TEST DATA OF THE SIX TASKS

\begin{tabular}{l|c|c|c|c|c}
\hline Tasks & $\begin{array}{c}\text { Training } \\
\text { (Positives:Negatives) }\end{array}$ & $\begin{array}{c}\text { Test } \\
\text { (Positives:Negatives) }\end{array}$ & $\begin{array}{c}\text { Event } \\
\text { Size }\end{array}$ & $\begin{array}{c}\text { Numbers of } \\
\text { Attributes } \\
\text { (No Features) }\end{array}$ & $\begin{array}{c}\text { Numbers of } \\
\text { Attributes } \\
\text { (Features) }\end{array}$ \\
\hline \hline 1. Single Point Changes in [-0.5,0.5] & $214: 185$ & $98: 101$ & 2 & 2 & \\
2. Single Point Changes in [-2.5,5.0] & $171: 228$ & $97: 102$ & 2 & 2 & 1 \\
3. Perturbed Periodical Function & $111: 282$ & $56: 137$ & 8 & 8 & 1 \\
4. Changes in Both Variables & $200: 199$ & $103: 96$ & 2 & 4 & 2 \\
5. Changes in Any Two out of Five & $193: 206$ & $110: 89$ & 2 & 10 & 5 \\
6. Video Shot Detection & $134: 482$ & $83: 902$ & 2 & 8 & 4 \\
\hline
\end{tabular}

TABLE IV

GP RUNTIME PARAMETERS

\begin{tabular}{l|r}
\hline Population & 50 \\
Generation & 30 \\
Maximum Depth & 5 \\
Minimum Depth & 2 \\
Mutation Rate & $5 \%$ \\
Crossover Rate & $85 \%$ \\
Elitism Rate & $10 \%$ \\
\hline
\end{tabular}

used to combine multiple classifiers as an ensemble to boost accuracy [20]. For each task, the best conventional classifier from the four was selected as the base classifier in AdaBoost.

For conventional classifiers, we manually segment raw data according to the event time span. Figure 9 shows how a twovariable time series is converted into a four-attribute learnable dataset when the event window size should be two. By such a conversion, each instance would contain all the variable values for one event span and nothing more. The optimal event window size is actually given through this data. Video was converted differently as there are simply too many attributes to be included in an instance, since the frame size is as high as $140 \times 80$. Instead of raw pixels, the average intensity, and the averages of $R, G, B$ values were fed into those conventional classifiers. The last three columns of Table $\mathrm{V}$ show the time span of each event, the numbers of attributes in the converted training data in raw values, and the numbers of attributes in the training data in extracted features.

Table VI lists all the results from the six methods applying on the same raw data of the six tasks. The results from our GP approach are listed at the rightmost column. All the results in the table were obtained from the test. Other than the test accuracy, the true positive rate (TP) and the true negative rate $(\mathrm{TN})$ are also listed for each case. It is clear that without pre-defined features, none of the five conventional methods used this study could achieve perfect or near perfect performance on any of the problems, not even the simplest single point change problem. On the perturbed function, IB1 managed to reach $95.34 \%$ of accuracy with good TP and $\mathrm{TN}$ rates. However it performed poorly on other problems. On the shot detection problem, the accuracies from the five conventional methods were not low. However that was not genuinely good performance as we can see their TP rates were all very low, even as low as zero. The high accuracies were caused by the unbalanced data of this task. By classifying everything to the majority class, a classifier could obviously

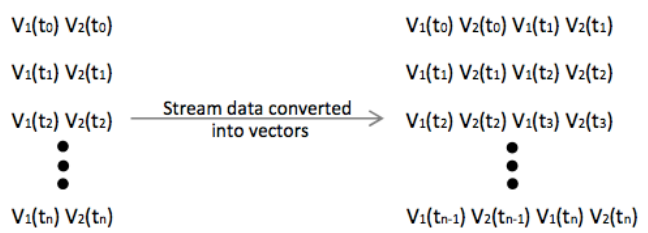

Fig. 9. Converting Raw Data Streams To Vectors for Conventional Classifiers

obtain a good accuracy although it is not very meaningful. In contrast with the poor results from other five methods, GP was perfect on five problems and was nearly perfect on the most difficult video shot detection problem. The good performance of GP seemingly was not affected by the absence of features, the inconsistency in the event size, the existence of multiple variables and even the spatial dependences among a large collection of variables. GP was significantly better than the five conventional methods on raw time series data points.

Similar to Table VI, Table VII presents the results of conventional methods using pre-defined features which are described in Section IV. The results of GP listed in the rightmost column are identical to those in Table VI. No feature was involved in GP runs. With the presence of features, other methods could achieve accuracies similar to those from GP. IB1 and AdaBoot achieved 100\% accuracy on five tasks and good accuracy on video shot detection. We can see that GP without pre-defined features can still achieve accuracies at least comparable to that from conventional methods. One point to emphasize is that the manually designed features for these six tasks were different to each other. A new problem likely needs a different set of features.

\section{Discussion AND ANALYSIS}

During the experiments, GP was able to find perfect solutions and terminated early around the 6th to 24th generations for the most of the problems. The result was promising even on tasks as complex as shot detection. This suggests that the representation mentioned in Section III is sufficient for detecting target events and solutions could be found quickly by such representation.

Some of the best individuals were analysed to reveal their behaviours. It would be interesting to learn what kind of features were obtained by the GP detector. Also good interpretability of the evolved solutions would increase the applicability of GP to real world tasks, especially these high- 
TABLE VI

Comparing With Conventional Methods on RaW Data \% (NO PRE-Defined Temporal Features)

\begin{tabular}{|c|c|c|c|c|c|c|}
\hline Tasks & OneR & $\mathrm{J} 48$ & NB & IB1 & AdaBoost & GP \\
\hline \multirow{3}{*}{ 1. Single Point Changes in $[-0.5,0.5]$} & 52.76 & 49.25 & 477.24 & 262.81 & 261.31 & $\overline{100}$ \\
\hline & ТP : 83.7 & TP : 100 & TP : 92.90 & TP : 79.60 & TP $: 81.60$ & TP : 100 \\
\hline & $\mathrm{TN}: 22.8$ & $\mathrm{TN}: 0$ & $\mathrm{TN}: 3.00$ & $\mathrm{TN}: 46.50$ & $\mathrm{TN}: 41.60$ & TN : 100 \\
\hline \multirow{3}{*}{ 2. Single Point Changes in $[-2.5,5.0]$} & 56.78 & 51.25 & 51.26 & 67.34 & 67.34 & 100 \\
\hline & ТP : 52.6 & TP : 0 & TP : 0 & TP : 68.00 & TP : 68.00 & TP : 100 \\
\hline & $\mathrm{TN}: 60.8$ & TN : 100 & TN $: 100$ & TN : 66.7 & TN : 66.7 & TN : 100 \\
\hline \multirow{3}{*}{ 3. Perturbed Periodical Function } & 70.98 & 70.98 & 70.98 & 95.34 & 94.82 & 100 \\
\hline & TP : 0 & TP : 0 & TP : 0 & TP $: 100$ & TP : 100 & TP : 100 \\
\hline & $\mathrm{TN}: 100$ & TN $: 100$ & TN : 100 & TN : 93.4 & TN : 92.7 & $\mathrm{TN}: \mathbf{1 0 0}$ \\
\hline \multirow{3}{*}{ 4. Changes in Both Variables } & 52.26 & 51.76 & 49.75 & 51.76 & 50.75 & 100 \\
\hline & TP : 44.70 & TP : 100 & TP $: 27.20$ & TP : 58.30 & TP : 58.30 & TP : 100 \\
\hline & TN : 60.4 & TN : 0 & TN : 74 & TN : 44.8 & $\mathrm{TN}: 42.7$ & TN : 100 \\
\hline \multirow{3}{*}{ 5. Changes in Any Two out of Five } & 47.73 & 55.28 & 57.29 & 50.25 & 57.29 & 100 \\
\hline & TP : 58.40 & TP : 0 & TP $: 10.10$ & TP : 48.30 & TP $: 10.10$ & TP : 100 \\
\hline & TN $: 39.10$ & TN $: 100$ & TN $: 95.5$ & $\mathrm{TN}: 51.8$ & TN : 95.5 & TN : 100 \\
\hline \multirow{3}{*}{ 6. Video Shot Detection } & 89.76 & 91.48 & 91.48 & 96.15 & 95.54 & 98.47 \\
\hline & $\mathrm{TP}: 4.80$ & $\mathrm{TP}: 0$ & $\mathrm{TP}: 0$ & TP $: 60.70$ & TP $: 58.30$ & TP : 97.59 \\
\hline & TN : 97.7 & $\mathrm{TN}: 100$ & $\mathrm{TN}: 100$ & $\mathrm{TN}: 99.40$ & TN : 99.00 & TN : 98.55 \\
\hline
\end{tabular}

TABLE VII

Comparing with Conventional Methods on Pre-defined Temporal Features \%

\begin{tabular}{|c|c|c|c|c|c|c|}
\hline Tasks & OneR & $\mathrm{J} 48$ & NB & IB1 & AdaBoost & GP \\
\hline \multirow{3}{*}{ 1. Single Point Changes in $[-0.5,0.5]$} & 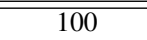 & 99.00 & 96.99 & 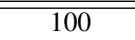 & 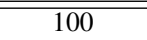 & $\overline{100}$ \\
\hline & TP $: 100$ & TP : 98.00 & TP : 93.90 & TP : 100 & TP : 100 & $\mathrm{TP}: \mathbf{1 0 0}$ \\
\hline & TN $: 100$ & TN : 100 & $\mathrm{TN}: 100$ & TN $: 100$ & $\mathrm{TN}: 100$ & $\mathrm{TN}: \mathbf{1 0 0}$ \\
\hline \multirow{3}{*}{ 2. Single Point Changes in $[-2.5,5.0]$} & 100 & 100 & 86.93 & 100 & 100 & 100 \\
\hline & TP : 100 & TP : 100 & TP : 73.2 & TP : 100 & TP : 100 & TP : 100 \\
\hline & TN $: 100$ & $\mathrm{TN}: 100$ & $\mathrm{TN}: 100$ & TN $: 100$ & $\mathrm{TN}: 100$ & TN : 100 \\
\hline \multirow{3}{*}{ 3. Perturbed Periodical Function } & 100 & 100 & 99.48 & 100 & 100 & 100 \\
\hline & TP $: 100$ & TP $: 100$ & TP $: 100$ & TP : 100 & TP $: 100$ & TP : 100 \\
\hline & $\mathrm{TN}: 100$ & $\mathrm{TN}: 100$ & TN : 99.30 & TN $: 100$ & $\mathrm{TN}: 100$ & TN : 100 \\
\hline \multirow{3}{*}{ 4. Changes in Both Variables } & 78.89 & 100 & 100 & 100 & 100 & 100 \\
\hline & TP $: 56.30$ & TP : 100 & TP : 100 & ТP : 100 & TP : 100 & TP : 100 \\
\hline & TN : 99.10 & $\mathrm{TN}: 100$ & $\mathrm{TN}: 100$ & TN $: 100$ & $\mathrm{TN}: 100$ & $\mathrm{TN}: \mathbf{1 0 0}$ \\
\hline \multirow{3}{*}{ 5. Changes in Any Two out of Five } & 65.82 & 97.99 & 87.94 & 100 & 100 & 100 \\
\hline & TP : 56.20 & ТP : 97.80 & ТP : 100 & TP : 100 & TP : 100 & TP : $\mathbf{1 0 0}$ \\
\hline & $\mathrm{TN}: 73.60$ & $\mathrm{TN}: 98.20$ & $\mathrm{TN}: 78.20$ & $\mathrm{TN}: 100$ & $\mathrm{TN}: 100$ & $\mathrm{TN}: \mathbf{1 0 0}$ \\
\hline \multirow{3}{*}{ 6. Video Shot Detection } & 95.94 & 96.75 & 98.68 & 98.17 & 98.68 & 98.47 \\
\hline & TP : 91.6 & TP : 98.8 & TP : 95.20 & TP : 92.80 & TP : 95.20 & ТР : 97.59 \\
\hline & TN : 96.30 & TN : 96.60 & TN : 99.00 & TN : 98.70 & $\mathrm{TN}: 99.00$ & TN : $\mathbf{9 8 . 5 5}$ \\
\hline
\end{tabular}

risk applications. To simplify the understanding, rather than the solutions evolved by GP, we represent them as equivalent programs which are more meaningful.

For the first task, "single point change", one of the best individual can be expressed as the following:

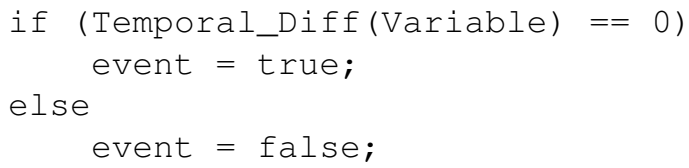

We can see that it simply calculates the difference between two consecutive points of the variable. A difference value within $[-0.5,0.5]$ would be treated as 0 . This GP detector found the exact feature for this event.

A example for the multivariable task No. 4 is shown below:

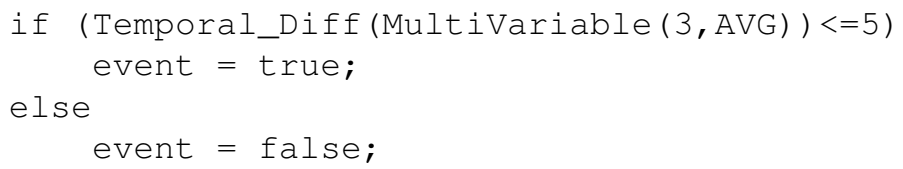

This program says that if the change of the average of the two variable is larger than 5 then it reports an event. The parameter 3 of function "MultiVariable" is 00000011 in binary. Therefore the last two variables, and the only two, are selected. So the characteristics of the data is indeed found by this GP detector. The evolved programs for multivariable problems are generally more complex.

However for perturbed periodic function and random numbers the evolved solutions are even more complex. That in a way reflects the difficulty of the problem. One of the best individuals which is also relatively small is such:

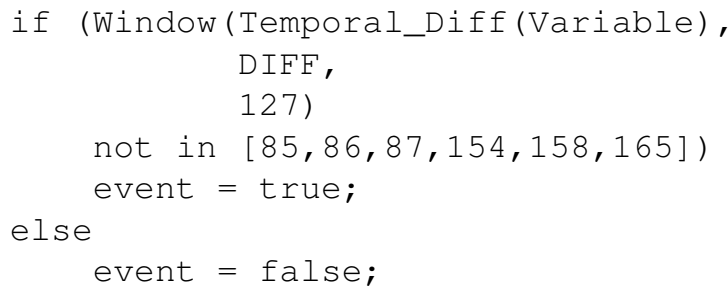


Compared to the above two programs, this program is more difficult to explain due to the higher complexity of function "Window" compared to "Temporal_Diff" function. This function "Window" takes the output of "Temporal_Diff()" as the first parameter, and treats it as its input time series. The second parameter 127 in binary form is 01111111 . So this function requires the last seven values from the "Temporal_Diff()" function. To generate these seven values, function "Temporal_Diff" must sample eight points from the original time series "Variable", which is the perturbed sine wave. The 8 points specified by this program is exactly the event size of that sine wave. This solution did successfully find the correct event size, although the exact operation of it is not entirely clear.

The evolved programs in video shot detection task are the least interpretable. What we can see is that the best individual only takes small proportion of the frame as the input. The three areas identified by it are area $(71,23)$ starting from coordinate $(94,4)$, area $(53,43)$ starting from coordinate $(94,4)$ and area $(71,23)$ starting from coordinate $(3,76)$. This detector essentially only requires 1978 points out of all 11200 pixels to achieve high detection accuracy. More details of this detector will be discussed separately.

\section{CONCLUSion AND Future WORK}

This study presented a GP based framework for event detection in time series. The framework uses tree based programs with a number of specialised operators for time series streams. A number of detection tasks of varying difficulty were used to evaluate the performance of the proposed method, some with a single variable and some with several variables. The results show that on raw data input, the GP method achieved perfect or near perfect detection performance on all six tasks. In comparison, five conventional classification methods performed poorly. Furthermore when temporal features were made available to these classifiers, the performance of the raw input based GP was still superior. Analysis of the evolved GP programs revealed that it was possible to extract human understandable forms of the decision making and, in fact, the programs had captured the regularities injected into the synthetic time series. The success in achieving high detection rates is not by chance.

In the current work, the maximum window size has been set to eight. This constraint would limit its application as a larger size may be required for more complex data such as weather data. In the near future we will introduce a mechanism for expanding the sliding window whenever necessary during the evolutionary process so that the framework can be evaluated on more tasks and may even be able to find better solutions for the current tasks.

\section{REFERENCES}

[1] R. Poli and W. Langdon and N. McPhee, A Field Guide to Genetic Programming, Lulu.com, 2008

[2] J. R. Koza, Genetic programming I:On the programming of computers by means of natural selection, MIT press, 1996
[3] J. Lohn, G. Hornby, D. Linden,An Evolved Antenna for Deployment on Nasa's Space Technology 5 Mission, Genetic Programming Theory and Practice II, pp. 301-315, Springer(2004)

[4] M. A. Kaboudan, Spatiotemporal Forecasting of Housing Price By Use of Genetic Programming, the 16th Annual Meeting of the Association of Global Business, 2004

[5] A. Song and B. Pinto, Study of GP representations for motion detection with unstable background, IEEE Congress on Evolutionary Computation, 2010

[6] M. Hetland and P. Strom, Temporal Rule Discovery using Genetic Programming and Specialised Hardware, Proceedings of the 4th International Conference on Recent Advances in Soft Computing, 2002

[7] N. Wagner and Z. Michalewicz, An analysis of adaptive windowing for time series forecasting in dynamic environments: further tests of the DyFor GP model, pp.1657-1664, Proceedings of the 10th annual conference on Genetic and evolutionary computation, 2008

[8] A. Song and F. Xie,Evolving automatic frame splitters,Proceedings of the 13th annual conference companion on Genetic and evolutionary computation, 2011

[9] R. C. Holte, Very simple classification rules per- form well on most commonly used datasets, vol. 11, pp.6391, Machine Learning, 1993

[10] R. Quinlan, C4.5: Programs for Machine Learning, Morgan Kaufmann Publishers, 1993

[11] George H. J, Pat L., Estimating Continuous Distributions in Bayesian Classifiers, pp.338345, Eleventh Conference on Uncertainty in Artificial Intelligence, 1995

[12] D. Aha, D. Kibler, Instance-based learning algorithms, vol. 6, pp.3766, Machine Learning, 1991

[13] Y. Freund, E. S. Robert, Experiments with a new boosting algorithm, pp.148156, Thirteenth International Conference on Machine Learning, 1996

[14] V. Guralnik and J. Srivastava, Event detection from time series data, pp.33-42, Proceedings of the fifth ACM SIGKDD international conference on Knowledge, 1999

[15] J. Hunter and N. McIntosh, Knowledge-based event detection in complex time series data, pp.271-280, Artificial Intelligence in Medicine,1999

[16] D. Preston and P. Protopapas and C. Brodley, Event discovery in time series, pp.61-72, Proceedings of the SIAM International Conference on Data Mining, 2009

[17] E. Keogh and J. Lin and A. Fu, Hot sax: Efficiently finding the most unusual time series subsequence, Fifth IEEE International Conference on Data Mining, 2005

[18] C. A. Ratanamahatana and J. Lin and D. Gunopulos and E. Keogh and M. Vlachos and G. Das, Mining time series data, Data Mining and Knowledge Discovery Handbook 2010, pp. 1049-1077, 2010

[19] C. Shahabi and D. Yan, Real-time pattern isolation and recognition over immersive sensor data streams, pp. 93-113, Proceedings of The 9th Intl Conference on Multi-Media Modeling, 2003

[20] F. Yoav, E. S.Robert, Experiments with a new boosting algorithm, pp.148-156,Thirteenth International Conference on Machine Learning, 1996

[21] L. Wei and E. Keogh, Semi-supervised time series classification, pp. 748753 , Proceedings of the 12th ACM SIGKDD international conference on Knowledge discovery and data mining, 2006

[22] A. Song, V. Ciesielski, Texture Segmentation by Genetic Programming, Evolutionary Computation, pp. 461-481, 2008

[23] T. Loveard, V. Ciesielski, Representing classification problems in genetic programming, Proceedings of the 2001 Congress on Evolutionary Computation, pp. 1070-1077, 2001 